\title{
The Lüders Band Problem
}

\section{T. Y. THOMAS}

1. Introduction. If a flat plate, such as is commonly used in the ordinary tensile test, is subjected to a slowly increasing load a Lüders band, represented by one of the set of inclined parallel lines in Fig. 1, will suddenly appear when

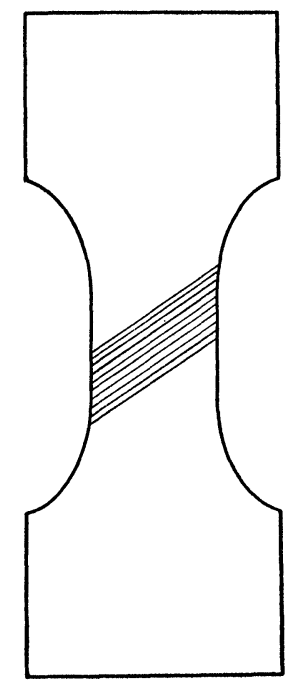

Frgure 1

the tension in the plate reaches the yield stress of the material. ${ }^{1}$ A slight decrease in the load ${ }^{2}$ will occur at the instant of formation of the band. With increasing

'Several years ago we presented an equilibrium theory of the Lüders band problem involving the interpretation of the velocity components in the plastic stress-strain relations as plastic displacements in accordance with usual engineering practice. It is the purpose of the present article to treat this problem from the strict dynamical viewpoint. See, T. Y. Thomas, On the inclination of plastic slip bands in flat bars in tension tests, Proc. N.A.S., 39 (1953) pp. 257-265; also, The effect of compressibility on the inclination of plastic slip bands in flat bars, ibid., pp. 266-273. In this connection reference may also be made to A. NADAI, Theory of Flow and Fracture of Solids, McGraw-Hill, New York, N.Y. (1950) pp. 320-327; R. HrLL, On discontinuous plastic states with special reference to localized necking in thin sheets, Jour. of Mech. and Phys. of Solids, 1 (1952) pp. 19-30; and G. R. IRwin, Angle and strain relations in flat plate Lüders bands, Jour. Appl. Mech., 20 (1953) pp. 449-450.

${ }^{2}$ See, T. Y. Thomas, $A$ discussion of the load drop and related matters associated with the 
load a second band will be formed, parallel and usually adjacent to the first, and this process can be continued until the plate fractures or is completely covered with these bands.

Certain general assumptions will be made as the basis for the following investigation. $^{3}$ In the first place we shall assume that the Lüders band consists of a thin strip of plastic material between two parallel planes, these planes being perpendicular to the flat sides of the plate. On each side of the band the material will be assumed to be in the elastic state and under uniform tension. If one end of the plate is in motion due to the applied load, after formation of the band, it will be assumed that the elastic region on the side of the band containing the moving end of the plate is displaced in a rigid manner. From the nature of the applied load this displacement must obviously be a translation and hence the velocity of the material particles in the elastic region will be the same at any instant. This implies, in particular, that if one end of the plate is clamped so as to retain a fixed position the elastic region on the side of the clamped end will remain at rest. Within the plastic region of the Lüders band the dynamical behavior of the particles will be subject to the plasticity equations which will later be introduced into the discussion.

Let us choose a fixed rectangular coordinate system $x$ such that the $x_{i}, x_{2}$ plane is parallel to the flat side of the plate and the $x_{2}$ axis is in the direction of the applied load (see Fig. 2). The $x_{3}$ axis will then be perpendicular to the flat

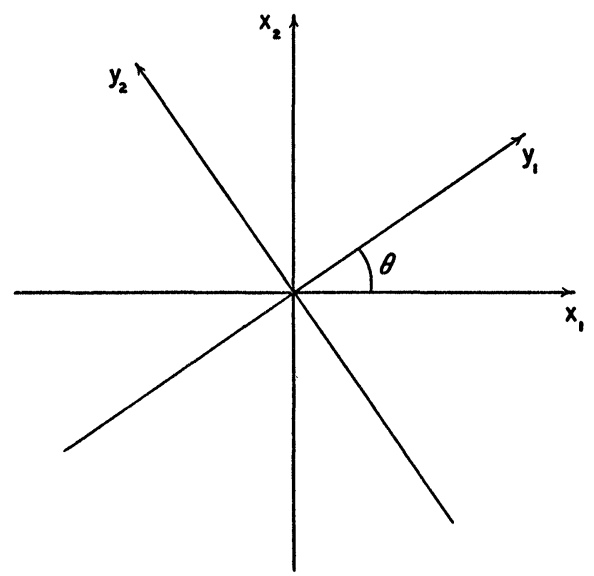

Figure 2

formation of a Lüders band, Proc. N.A.S., 40 (1954) pp. 572-576. In the present article it is assumed that the band has been formed and we are concerned primarily with the dynamical relationships which must exist along the planes of separation of the plastic material in the Lüders band and the surrounding elastic material of the plate.

${ }^{3}$ This investigation was conducted at the request of the Naval Research Laboratory and has been supported by the Office of Naval Research under Contract Nonr-908(09), Indiana University, NR 041037. 
side of the plate. We shall assume that the plate is in a state of plane stress which is justified since its thickness is small in comparison with its length and width. Then if $\sigma_{i k}$ are the components of the symmetric stress tensor relative to the $x$ system, or the $y$ coordinate system which will be introduced in $\S 3$, we have

$$
\sigma_{i k}=f_{i k}\left(x_{1}, x_{2}, t\right) ; \quad \sigma_{i 3}=0,
$$

where the f's are functions of the two coordinates $x_{1}, x_{2}$ and the time $t$ alone. Conditions (1) will be beneficial in treating the plastic material within the Lüders band. In the elastic regions surrounding the Lüders band the components $\tau_{i k}$ of the stress tensor, relative to the $x$ system, are given simply by $\tau_{22}=\tau$ and $\tau_{i k}=0$ otherwise, where $\tau$ is the yield stress of the material.

One frequently speaks of the planes bounding the Lüders band as slip planes. Precisely, this means that the condition

$$
\left[v_{i}\right] v_{i}=0
$$

is satisfied where $\nu_{i}$ are the components of the unit normal vector to the planes and $\left[v_{i}\right]$ denotes the difference in the values of the velocity components at adjacent points on the two sides of these planes relative to some dynamically allowable coordinate system. For definiteness we specify that the vector $\nu$ is directed from the plastic to the elastic side of the slip plane under consideration and that the difference $\left[v_{i}\right]=\bar{v}_{i}-w_{i}$ where the $\bar{v}_{i}$ are the values, at points of the slip plane, of the velocity components $v_{i}$ in the Lüders band; also $w_{i}$ are the velocity components in the elastic region outside the band, these components being independent of position but not necessarily independent of the time by the above assumptions. A similar designation will be employed in the following discussion. Thus the use of a bar over a quantity or its derivative will denote evaluation on the slip plane.

2. Dynamical conditions. Let $\Sigma$ denote the slip plane bounding one side of the Lüders band. Then over $\Sigma$ we have the following dynamical relations

$$
\begin{gathered}
\rho\left(G-w_{n}\right)=\bar{\rho}\left(G-\bar{v}_{n}\right), \\
{\left[\sigma_{i j}\right] \nu_{i}=-\rho\left(G-w_{n}\right)\left[v_{i}\right],}
\end{gathered}
$$

which are valid in any allowable coordinate system. In these relations $\rho$ and $w_{n}$ denote the density and the normal component of the velocity of the material particles on the elastic side of $\Sigma$. The corresponding barred quantities $\bar{\rho}$ and $\bar{v}_{n}$ denote density and normal velocity in the plastic strip adjacent to $\Sigma$ as explained in $\$ 1$. Also $G$ is the normal coordinate velocity of the slip plane in the direction of its normal $\nu$ and $\left[\sigma_{i k}\right]=\bar{\sigma}_{i k}-\mu_{i k}$ where $\bar{\sigma}_{i k}$ are the values of the components $\sigma_{i k}$ of the stress tensor in the plastic band at points of $\Sigma$ while the $\mu_{i k}$ are the components of the stress tensor in the elastic region on the other side of $\Sigma$. 
It is seen immediately that $w_{n}=\bar{v}_{n}$ from (2). Then the difference $G-w_{n}$ or $G-\bar{v}_{n}$ is called the speed of propagation of $\Sigma$. Assuming that this speed is equal to zero it follows that (3) is satisfied automatically and (4) reduces to

$$
\left[\sigma_{i k}\right] \nu_{k}=\bar{\sigma}_{i k} \nu_{k}-\mu_{i k} \nu_{k}=0 \text {. }
$$

3. Rotation of axes. By rotating the $x_{1}$ and $x_{2}$ axes through an angle $\theta$ about the $x_{3}$ axis we can introduce a new coordinate system $y$ for which the $y_{1}$ axis will be parallel to the plane $\Sigma$ and the $y_{2}$ axis will be perpendicular to $\Sigma$. This is illustrated in Fig. 2. The coordinates $x$ and $y$ of these two systems will be related by the following transformation

$$
x_{1}=y_{1} \cos \theta-y_{2} \sin \theta ; \quad x_{2}=y_{1} \sin \theta+y_{2} \cos \theta ; \quad x_{3}=y_{3} .
$$

The components $\mu_{i k}$ of the stress tensor in the elastic region, relative to the $y$ system, are obtained from the components $\tau_{i k}$ in the $x$ system by the tensor law of transformation. Carrying out this operation we find that

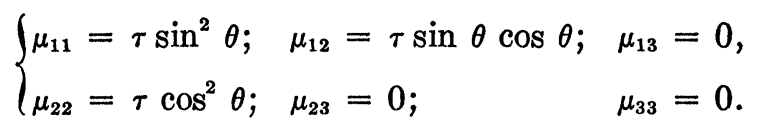

Use of the $y$ system, while not necessary, will be advantageous in the following discussion.

4. Conditions resulting from symmetry and the assumption of plastic incompressibility. Let $v_{i}$ denote the velocity components in the Lüders band relative to the $y$ system and $v_{i, k}$ the partial derivative of $v_{i}$ with respect to $y_{k}$. Then, neglecting possible end effects of the plate, we see immediately from symmetry that $v_{i, 1}=0$ within the plastic strip; hence $\bar{v}_{i, 1}=0$ in particular. Now

$$
G=\bar{v}_{n}=\bar{v}_{i} \nu_{i}=\bar{v}_{2},
$$

since the $\nu_{i}$ have the values $0,1,0$ in the $y$ system. But $G$ is constant on the slip plane $\Sigma$ at any time $t$ since $\Sigma$ is either stationary or is displaced into parallel planes. Hence $\bar{v}_{2}=$ const. and hence $\bar{v}_{2,3}=0$ at any time $t$.

Let us now put $\bar{v}_{i, 2}=\omega_{i}$ for $i=1,2,3$ and let us consider the usual condition of incompressibility of plastic material. Then at points adjacent to the slip plane $\Sigma$ we have

$$
\bar{v}_{i, i}=\bar{v}_{1,1}+\bar{v}_{2,2}+\bar{v}_{3,3}=0 .
$$

But the term $\bar{v}_{1,1}$ vanishes as observed above and hence the condition of incompressibility reduces to $\bar{v}_{3,3}=-\omega_{2}$. Also putting $\bar{v}_{1,3}=\omega$ we see that the quantities

The fact that the normal velocity $G$ of the slip plane is equal to the velocity $\bar{v}_{n}$ of the material particles shows that the band does not grow in the sense that new material is added to it but merely stretches under the influence of the applied load.

Conversely if we assume that the plastic band is capable of stretching only we see that $G=\bar{v}_{n}$. But $\bar{v}_{n}=w_{n}$ from (2) and hence it follows as above that (3) is satisfied and that (4) reduces to (5). 
$\bar{v}_{i, k}$ are given by the following equations

(7)

$$
\left\{\begin{array}{lll}
\bar{v}_{1,1}=0 ; & \bar{v}_{1,2}=\omega_{1} ; & \bar{v}_{1,}=\omega, \\
\bar{v}_{2,1}=0 ; & \bar{v}_{2,2}=\omega_{2} ; & \bar{v}_{2,3}=0, \\
\bar{v}_{3,1}=0 ; & \bar{v}_{3,2}=\omega_{3} ; & \bar{v}_{3,3}=-\omega_{2} .
\end{array}\right.
$$

The quantities $\epsilon_{i k}$ defined by

$$
\epsilon_{i k}=\frac{1}{2}\left(v_{i, k}+v_{k, i}\right)
$$

are the components of the rate of strain tensor. Using the above equations (7) for the $\bar{v}_{i, k}$ we therefore have

$$
\left\{\begin{array}{lll}
\bar{\epsilon}_{11}=0 ; & \bar{\epsilon}_{12}=\frac{\omega_{1}}{2} ; & \bar{\epsilon}_{13}=\frac{\omega}{2} \\
\bar{\epsilon}_{22}=\omega_{2} ; & \bar{\epsilon}_{23}=\frac{\omega_{3}}{2} ; & \bar{\epsilon}_{33}=-\omega_{2} .
\end{array}\right.
$$

5. The von Mises stress-strain relations. The stress-strain relations of von Mises for plastic material are

$$
\epsilon_{i k}=\psi \sigma_{i k}^{*}, \quad \psi=\frac{\epsilon_{i k} \sigma_{i k}^{*}}{K}
$$

where $K>0$ is a material constant and the $\sigma_{i k}^{*}$ are the components of the stress deviator which is defined in terms of the stress components $\sigma_{i k}$ by the equations

$$
\sigma_{i k}^{*}=\sigma_{i k}+p \delta_{i k}, \quad p=-\frac{1}{3} \sigma_{i i} .
$$

It is assumed that the proportionality factor $\psi$ in (9) is positive and finite.

If we multiply both members of (9) by $\sigma_{i k}^{*}$ and sum on the repeated indices $i$ and $k$ we find that

$$
K=\sigma_{i k}^{*} \sigma_{i k}^{*} .
$$

This condition, which is a consequence of the form (9) of the stress-strain relations, is usually referred to as the quadratic yield condition.

From (8), (9) and (10) we now have

$$
\left\{\begin{array}{lll}
\bar{\sigma}_{11}=-\bar{p} ; & \bar{\sigma}_{12}=\frac{\omega_{1}}{2 \bar{\psi} ;} & \bar{\sigma}_{13}=\frac{\omega}{2 \bar{\psi}} \\
\bar{\sigma}_{22}=\frac{\omega_{2}}{\bar{\psi}}-\bar{p} ; & \bar{\sigma}_{23}=\frac{\omega_{3}}{2 \bar{\psi} ;} & \bar{\sigma}_{33}=-\frac{\omega_{2}}{\bar{\psi}}-\bar{p} .
\end{array}\right.
$$

It follows from these relations and the plane stress condition (1) that $\omega=\omega_{3}=0$. Also we see from the last of the above equations that

$$
\omega_{2}+\bar{p} \bar{\psi}=0 \text {. }
$$


Making use of (12) the non-vanishing quantities $\bar{\sigma}_{i k}$ are therefore given by

$$
\bar{\sigma}_{11}=-\bar{p} ; \quad \bar{\sigma}_{12}=\frac{\omega_{1}}{2 \bar{\psi}} ; \quad \bar{\sigma}_{22}=\frac{2 \omega_{2}}{\bar{\psi}} .
$$

6. Inclination of the Liiders bands. Relative to the $y$ system the conditions (5) yield

$$
\bar{\sigma}_{i 2}=\mu_{i 2} \text {. }
$$

These relations are satisfied identically for $i=3$. However, taking $i=1$ and 2 and making use of (6) and (13), the relations (14) give

$$
\omega_{1}=2 \tau \bar{\psi} \sin \theta \cos \theta ; \quad 2 \omega_{2}=\tau \bar{\psi} \cos ^{2} \theta .
$$

Eliminating the quantity $\tau \bar{\psi}$ from the equations (15) we find that

$$
\tan \theta=\frac{\omega_{1}}{4 \omega_{2}}
$$

The angle $\theta$ can also be eliminated from the two relations (15). This gives

$$
\tau \bar{\psi}=\frac{\omega_{1}^{2}+16 \omega_{2}^{2}}{8 \omega_{2}} .
$$

Let us now turn our attention to the yield condition (11). Expand the right member of (11) and make the substitution (10). Then put $\sigma_{13}=\sigma_{23}=\sigma_{33}=0$ in accordance with the plane stress condition. Finally evaluate at points in the plastic band adjacent to the slip plane $\Sigma$ and make the substitution (13) for the remaining components $\sigma_{i k}$. We thus obtain the equation

$$
\left(\frac{2 \omega_{2}}{\bar{\psi}}+\bar{p}\right)^{2}+\frac{\omega_{1}^{2}}{2 \bar{\psi}^{2}}+\bar{p}^{2}=K
$$

or

$$
\frac{\omega_{1}^{2}}{2 \bar{\psi}^{2}}+\frac{2 \omega_{2}^{2}}{\bar{\psi}^{2}}=K
$$

when we eliminate $\bar{p}$ by means of (12). But it is seen that $K=\frac{2}{3} \tau^{2}$ from the invariant relation (11) when we use the values $\tau_{22}=\tau$ and $\tau_{i k}=0$ (otherwise) of the components of the stress tensor in the elastic region relative to the $x$ coordinate system. Hence (18) becomes

$$
4 \tau^{2} \bar{\psi}^{2}=3 \omega_{1}^{2}+12 \omega_{2}^{2}
$$

Since $\tau$ is positive and $\psi$ is also positive by the assumption of $\$ 5$, the equation (19) is equivalent to the relation

$$
2 \tau \bar{\psi}=\sqrt{3 \omega_{1}^{2}+12 \omega_{2}^{2}} .
$$

Eliminating the quantity $\tau \bar{\psi}$ between the two equations (17) and (20) the resulting relation in $\omega_{1}$ and $\omega_{2}$ readily yields $\omega_{1} / \omega_{2}= \pm 2 \sqrt{2}$. Hence (16) becomes 


$$
\tan \theta= \pm \frac{1}{\sqrt{2}} \cdot
$$

Hence there are two families of parallel Lüders bands which have inclinations of $35^{\circ} 16^{\prime}$ and $-35^{\circ} 16^{\prime}$ respectively with the $x_{1}$ axis. These two families of bands are observed in the tension test described at the beginning of $\$ 1$.

7. Prandtl-Reuss equations. We shall now consider the Lüders band problem when the von Mises equations (9) are replaced by the Prandtl-Reuss equations for the determination of plastic flow. These latter equations are frequently written in the following form

$$
\frac{d \sigma_{i k}^{*}}{d t}=2 \mu\left(\epsilon_{i k}-\psi \sigma_{i k}^{*}\right),
$$

where $\mu$ is the usual elastic constant and the other quantities in the equations have been defined in the foregoing sections. We observe that the discussion and results preceding $\S 5$ remain valid since they are independent of the particular form of the stress-strain relations. Our specific task is now to obtain algebraic relations between the components of the stress and rate of strain tensors which will lead to a determination of the inclination $\theta$ of the Lüders bands in spite of the complication caused by the fact that the above equations (22) involve derivatives of the stress components.

Let us first take the $\delta$ time derivative ${ }^{5}$ of the equations (5). This gives

$$
\left[\frac{\delta \sigma_{i k}}{\delta t}\right] \nu_{k}=\frac{\delta \bar{\sigma}_{i k}}{\delta t} \nu_{k}=0
$$

since $\delta \nu_{k} / \delta t=0$ and also $\delta \mu_{i k} / \delta t=0$ due to the fact that the components of the stress tensor are constant in the elastic region. But $G=\bar{v}_{2}$ and $\nu_{k}=(0,1,0)$ relative to the $y$ system. Hence

$$
\frac{\delta \bar{\sigma}_{i k}}{\delta t}=\frac{\partial \bar{\sigma}_{i k}}{\partial t}+\bar{\sigma}_{i k, m} G \nu_{m}=\frac{\partial \bar{\sigma}_{i k}}{\partial t}+\bar{\sigma}_{i k, 2} \bar{v}_{2} .
$$

Now consider the relations

$$
\frac{d \sigma_{i k}}{d t}=\frac{\partial \sigma_{i k}}{\partial t}+\sigma_{i k, m} v_{m},
$$

in the $y$ system. But $\sigma_{i k, 1}=0$ from symmetry and $\sigma_{i k, 3}=0$ from the plane stress condition. Hence (25) gives

$$
\frac{d \bar{\sigma}_{i k}}{d t}=\frac{\partial \bar{\sigma}_{i k}}{\partial t}+\bar{\sigma}_{i k, 2} \bar{v}_{2} .
$$

\footnotetext{
'T. Y. Thоmas, Extended compatibility conditions for the study of surfaces of discontinuity in continuum mechanics, Jour. Math. and Mech., 6 (1957) pp. 311-322.
} 
Comparing (24) and (26) we see that

$$
\frac{d \bar{\sigma}_{i k}}{d t}=\frac{\delta \bar{\sigma}_{i k}}{\delta t}
$$

In other words the $\delta$ time derivative of the component $\sigma_{i \text { i }}$ is equal to the total time derivative of this component at points in the plastic band adjacent to the slip plane $\Sigma$.

From (10), (23) and (27) we have

$$
\frac{d \bar{\sigma}_{i k}^{*}}{d t} \nu_{k}=\left(\frac{d \bar{\sigma}_{i k}}{d t}+\frac{d \bar{p}}{d t} \delta_{i k}\right) \nu_{k}=\frac{d \bar{p}}{d t} \nu_{i} .
$$

Hence from (22) and (28) it follows that

or

$$
\frac{d \bar{p}}{d t} \nu_{i}=2 \mu\left(\bar{\epsilon}_{i k}-\bar{\psi} \bar{\sigma}_{i k}^{*}\right) \nu_{k}
$$

$$
\frac{d \bar{p}}{d t} \delta_{i 2}=2 \mu\left(\bar{\epsilon}_{i 2}-\bar{\psi} \bar{\sigma}_{i 2}^{*}\right)
$$

relative to the $y$ system. Hence, taking $i=1,2,3$ respectively in (29), we find

$$
\begin{aligned}
& \bar{\epsilon}_{12}=\bar{\psi} \bar{\sigma}_{12}^{*}=\bar{\psi} \bar{\sigma}_{12}, \\
& \frac{d \bar{p}}{d t}=2 \mu\left(\bar{\epsilon}_{22}-\bar{\psi} \bar{\sigma}_{22}^{*}\right), \\
& \bar{\epsilon}_{23}=\bar{\psi} \bar{\sigma}_{23}^{*}=\bar{\psi} \bar{\sigma}_{23} .
\end{aligned}
$$

To deduce other relations which will be applicable to the problem at hand we take $i=1,2,3$ and $k=3$ in (22). This leads to the following set of three equations

$$
\begin{aligned}
& \bar{\epsilon}_{13}=\bar{\psi} \bar{\sigma}_{13}^{*}=\bar{\psi} \bar{\sigma}_{13}, \\
& \bar{\epsilon}_{23}=\bar{\psi} \bar{\sigma}_{23}^{*}=\bar{\psi} \bar{\sigma}_{23}, \\
& \frac{d \bar{p}}{d t}=2 \mu\left(\bar{\epsilon}_{33}-\bar{\psi} \bar{\sigma}_{33}^{*}\right) .
\end{aligned}
$$

Now equations (32) and (34) are identical; moreover $\bar{\sigma}_{23}=0$ in these equations and hence $\bar{\epsilon}_{23}=0$ or $\omega_{3}=0$ from (8). Similarly equation (33) reduces to $\omega=0$. Also eliminating $d \bar{p} / d t$ between (31) and (35) and using (10) and the plane stress condition we have

$$
\bar{\epsilon}_{22}-\bar{\psi} \bar{\sigma}_{22}=\bar{\epsilon}_{33} \text {. }
$$

Finally, when use is made of (8), the equations (30) and (36) give

$$
\omega_{1}=2 \bar{\psi} \bar{\sigma}_{12} ; \quad 2 \omega_{2}=\bar{\psi} \bar{\sigma}_{22} .
$$

The relations (14) are valid in the present discussion since they are merely the result of expressing (5) in the $y$ system. Hence (37) becomes

$$
\omega_{1}=2 \bar{\psi} \mu_{12}=2 \tau \bar{\psi} \sin \theta \cos \theta,
$$




$$
2 \omega_{2}=\bar{\psi} \mu_{22}=\tau \bar{\psi} \cos ^{2} \theta .
$$

when use is made of (6) and (14). These relations are the same as the previous relations (15) which were used in the determination of the angle $\theta$ in $\$ 6$.

Now observe that the relations (37) are identical with the second and third relation (13). To deduce the first relation (13) we can proceed as follows. Expand the right member of the yield condition (11), using (10), and evaluate at points in the plastic band lying on $\Sigma$. Replace the components $\bar{\sigma}_{12}$ and $\bar{\sigma}_{22}$ by the values given by (37) and put $\bar{\sigma}_{13}=\bar{\sigma}_{23}=\bar{\sigma}_{33}=0$ in accordance with the plane stress condition. This gives the relation

or

$$
\bar{\sigma}_{11}^{2}-\left(\frac{2 \omega_{2}}{\bar{\psi}}\right)_{\bar{\sigma}_{11}}+\frac{4 \omega_{2}^{2}}{\bar{\psi}^{2}}+\frac{3 \omega_{1}^{2}}{4 \bar{\psi}^{2}}=\frac{3}{2} K,
$$

$$
\bar{\sigma}_{11}^{2}-\left(\tau \cos ^{2} \theta\right) \bar{\sigma}_{11}+\tau^{2} \cos ^{4} \theta+3 \tau^{2} \sin ^{2} \theta \cos ^{2} \theta=\frac{3}{2} K,
$$

when use is made of (15). But this latter relation shows that the component $\sigma_{11}$ has a constant value on the plastic side of the surface $\Sigma$. Hence $\delta \bar{\sigma}_{11} / \delta t=0$ and hence $d \bar{\sigma}_{11} / d t=0$ from (27). Similarly $d \bar{\sigma}_{22} / d t=0$ since $\bar{\sigma}_{22}$ is constant on account of the relation

$$
\bar{\sigma}_{22}=\mu_{22}=\tau \cos ^{2} \theta,
$$

which follows from (6) and (14). Using these results we now see that $d \bar{\sigma}_{11}^{*} / d t=0$ and hence

$$
\bar{\epsilon}_{11}-\bar{\psi} \bar{\sigma}_{11}^{*}=-\bar{\psi} \bar{\sigma}_{11}^{*}=0,
$$

from (8) and (22). Hence, since $\bar{\psi} \neq 0$ by assumption, we have $\bar{\sigma}_{11}^{*}=0$ which is equivalent to the first relation (13). Finally, using the first relation (13), we can immediately deduce the relation (12).

We have now obtained the exact relations (12), (13) and (15) which were used in $\S 6$ to determine the inclination $\pm 35^{\circ} 16^{\prime}$ of the Lüders bands. Hence the Lïders bands have the same inclination regardless of whether the plastic flow is assumed to be governed by the Prandtl-Reuss or the von Mises stress-strain relations.

8. Modified Prandtl-Reuss equations. Let us now replace the ordinary or total time derivative of the components $\sigma_{i k}^{*}$ in the left members of (22) by the absolute time derivative $e^{6}$ of the stress deviator $\sigma^{*}$. We thus have

$$
\frac{D \sigma_{i k}^{*}}{D t}=2 \mu\left(\epsilon_{i k}-\psi \sigma_{i k}^{*}\right),
$$

where the quantities $D_{\sigma_{i k}^{*}}^{*} / D t$ are given by

$$
\frac{D \sigma_{i k}^{*}}{D t}=\frac{d \sigma_{i k}^{*}}{d t}+\sigma_{i m}^{*} \phi_{m k}+\sigma_{k m}^{*} \phi_{m i},
$$

oT. Y. Thomas, Kinematically preferred coordinate systems, Proc. N.A.S., 41 (1955) pp. 762-770. 
in which

$$
\phi_{i k}=\frac{1}{2}\left(v_{i, k}-v_{k, i}\right) .
$$

The equations (38) give the correct dynamical form of the Prandtl-Reuss stress-strain relations. ${ }^{7}$

The treatment of equations (38) is similar to the treatment of the equations (22) in \$7. Thus from (10), (38) and (39) we have

$$
\frac{d \sigma_{i k}}{d t}+\frac{d p}{d t} \delta_{i k}+\sigma_{i m} \phi_{m k}+\sigma_{k m} \phi_{m i}=2 \mu\left(\epsilon_{i k}-\psi \sigma_{i k}-\psi p \delta_{i k}\right),
$$

when use is made of the fact that the $\phi$ 's are skew-symmetric from (40). Now multiply (41) by $\nu_{k}$ and sum on the repeated index $k$; taking account of (23) and (27) we are led to the equations

$$
\frac{d \bar{p}}{d t} \delta_{i 2}+\bar{\sigma}_{i m} \bar{\phi}_{m 2}+\bar{\sigma}_{2 m} \bar{\phi}_{m i}=2 \mu\left(\bar{\epsilon}_{i 2}-\bar{\psi} \bar{\sigma}_{i 2}-\bar{\psi} \bar{p} \delta_{i 2}\right)
$$

which are valid in the $y$ coordinate system at points adjacent to the slip plane $\Sigma$. Putting $i=1,2,3$ respectively in (42), expanding, and using the plane stress condition (1) we obtain

$$
\left\{\begin{array}{c}
\bar{\sigma}_{11} \bar{\phi}_{12}+\bar{\sigma}_{22} \bar{\phi}_{21}=2 \mu\left(\bar{\epsilon}_{12}-\bar{\psi} \bar{\sigma}_{12}\right) \\
\frac{d \bar{p}}{d t}+2 \bar{\sigma}_{12} \bar{\phi}_{12}=2 \mu\left(\bar{\epsilon}_{22}-\bar{\psi} \bar{\sigma}_{22}-\bar{\psi} \bar{p}\right) \\
\bar{\sigma}_{12} \bar{\phi}_{13}+\bar{\sigma}_{22} \bar{\phi}_{23}=2 \mu \bar{\epsilon}_{23} .
\end{array}\right.
$$

Next take $k=3$ and $i=1,2,3$ in turn in the equations (41). Expanding and evaluating at points adjacent to the slip plane $\Sigma$ we now find that

$$
\left\{\begin{array}{c}
\bar{\sigma}_{11} \bar{\phi}_{13}+\bar{\sigma}_{12} \bar{\Phi}_{23}=2 \mu \bar{\epsilon}_{13} \\
\bar{\sigma}_{12} \bar{\phi}_{13}+\bar{\sigma}_{22} \bar{\Phi}_{23}=2 \mu \bar{\epsilon}_{23} \\
\frac{d \bar{p}}{d t}=2 \mu\left(\bar{\epsilon}_{33}-\bar{\psi} \bar{p}\right) .
\end{array}\right.
$$

If we neglect terms of the order of the small quantity $\tau / \mu$ in comparison with unity and avail ourselves of the relations (7) the first and second equations (44) become

$$
2 \mu \omega+\bar{\sigma}_{12} \omega_{3}=0 ; \quad \bar{\sigma}_{12} \omega-2 \mu \omega_{3}=0 .
$$

But from these relations it follows that $\omega=\omega_{3}=0$ as found in $\S 5$ and $\S 7$.

7See, C. Truesdeln, The simplest rate theory of pure elasticity, Comm. Pure and Appl. Math., 8 (1955) pp. 123-132; W. Nol,, On the continuity of the solid and fluid states, Jour. Rat. Mech. and Anal., 4 (1955) pp. 3-81. See also, T. Y. Thomas, On the structure of the stressstrain relations, Proc. N.A.S., 41 (1955) pp. 716-720, and Kinematically preferred coordinate systems, ibid. pp. 762-770. 
Eliminating $d \bar{p} / d t$ between the second equation (43) and the third equation (44) and combining the resulting equation with the first equation (43) we have

$$
\left\{\begin{array}{l}
\left(\bar{\sigma}_{11}-\bar{\sigma}_{22}\right) \bar{\phi}_{12}=2 \mu\left(\bar{\epsilon}_{12}-\bar{\psi} \bar{\sigma}_{12}\right), \\
\bar{\sigma}_{12} \bar{\phi}_{12}=\mu\left(\bar{\epsilon}_{22}-\bar{\epsilon}_{33}-\bar{\psi} \bar{\sigma}_{22}\right) .
\end{array}\right.
$$

Again neglecting quantities of the order $\tau / \mu$ or higher in comparison with unity, the first equation (45) gives $\bar{\epsilon}_{12}=\bar{\psi} \bar{\sigma}_{12}$. Hence, using (6), (8) and (14) we find

$$
\omega_{1}=2 \tau \bar{\psi} \sin \theta \cos \theta .
$$

Similarly the second equation (45) becomes

$$
\frac{1}{2} \omega_{1} \tau \sin \theta \cos \theta=\mu\left(2 \omega_{2}-\bar{\psi} \tau \cos ^{2} \theta\right) .
$$

Eliminating $\omega_{1}$ by means of (46) this relation can be written

$$
2 \omega_{2}=\left(1+\frac{\tau}{\mu} \sin ^{2} \theta\right) \tau \psi \cos ^{2} \theta,
$$

and hence reduces to

$$
2 \omega_{2}=\tau \bar{\psi} \cos ^{2} \theta,
$$

when the term involving the ratio $\tau / \mu$ is neglected in comparison with unity. We observe that the equations (46) and (47) are identical with the previous equations (15).

We shall now use the above equations (46) and (47) for the determination of the angle $\theta$ but the procedure by which this will be accomplished will be different from that employed at the end of $\S 7$. In fact, let us consider the relation

$$
\psi=\frac{\bar{\epsilon}_{i k} \bar{\sigma}_{i k}^{*}}{K}=\frac{\bar{\epsilon}_{i k} \bar{\sigma}_{i k}}{K},
$$

which is obtained when we multiply the equations (38) by $\sigma_{i k}^{*}$ and take account of the yield condition (11). Expanding the right member of (48) we obtain

$$
\bar{\psi}=\frac{\omega_{1} \mu_{12}+\omega_{2} \mu_{22}}{K},
$$

when use is made of the plane stress condition and the relations (8) and (14). Now put $K=\frac{2}{3} \tau^{2}$ and also make the substitutions (6) and (15) for the quantities appearing in the right member of (49). Then, since $\bar{\psi} \neq 0$, the resulting equation reduces to

$$
9 \cos ^{4} \theta-12 \cos ^{2} \theta+4=\left(3 \cos ^{2} \theta-2\right)^{2}=0 .
$$

Hence $\cos ^{2} \theta=\frac{2}{3}$. It follows that the Lüders bands also have the inclination $35^{\circ} 16^{\prime}$ or $-35^{\circ} 16^{\prime}$ when the plastic flow is governed by the modified Prandtl-Reuss equations (38). 